\title{
Line Flow Based WLAV State Estimation using BBO and WIPSO Techniques
}

\author{
M.Kalpanadevi ${ }^{1}$, R.Neela \\ ${ }^{I}$ (Assistant Professor, Dept. of Electrical Engg., Annamalai University, India) \\ ${ }_{2}^{2}$ (Professor, Dept. of Electrical Engg., Annamalai University, India)
}

\begin{abstract}
This paper presents a new line flow based weighted least absolute value (LFWLAV) technique for power systems making use of line flows, bus power injections and bus voltage magnitudes as the measurement vector has been suggested and it has been solved through BBO and WIPSO methods. State variables are estimated by means of constant, line flow based jacobian matrix developed from the network equations. The proposed selection of state variables has been advantageous as the jacobian matrix turns out to be constant which reduces the computational burden. Moreover, the suggested technique generates an output which gives a suggestion related to the overloaded lines and limit violated buses instantly rather than calculating them through separate equations. Weighted Least Squares (WLS) technique has been applied for solving the proposed line flow based state equations in the absence as well as presence of bad measurements and the results are validated against those obtained using the conventional WLAV technique. The effectiveness of the proposed method was examined by computer simulations through three test systems: (1) 14-bus IEEE test system, (2) 30bus IEEE test system and (3) 57-bus IEEE test system. Its convergence and calculation time had been determined cautiously and when compared with options acquired with the aid of the standard WLAV method. The results show that the proposed LFWLAV method spends less execution time than the standard method does with similar convergence characteristics.
\end{abstract}

Keywords : State Estimation, Weighted Least Absolute value method, Line flow based WLAV, LFWLAV-BBO, LFWLAV-WIPSO and Power System.

\section{Introduction}

State estimation assumes a significant part in the observing and control of present day power system. State estimation techniques are basically data processing algorithms which are applied on power systems to obtain the best estimate of current operating state from the available set of redundant measurements and network topology information. In 1968, Fred C. Schweppe introduced state estimation to the power system. The mathematical model and the general state estimation are explained in [1]. In [2] an approximate mathematical model and solution for detection and identification are mentioned. Different usage issues connected with dimensionality, computer speed, storage and the time-varying nature of actual power systems is additionally discussed in [3]. A technique for processing the measured data from electric power networks in order to obtain the best possible estimate of system variables. Making use of Taylor series and a least-squares criterion is outlined in [4]. A fast decoupled SE technique based on equivalent current injections and rectangular coordinates is discussed in (5). This actually is a break through technique as it resulted in identical sub gain matrices that needed to be updated and factorized only once. This technique, promising from the point of view of speed and applicability tends to generate a compromising estimate, by maintaining same weighting factors for both real and reactive components.

In order to solve SE problem, an alternative formulation of the state estimation problem, Weight Least Absolute Values (WLAV) has been used. The transformation-based WLAV estimator with leverage points are viewed in [6]. Leverage points are uniformly disbursed in the factor space of multiple regressions through linear transformations. This transformed system of measurement equations is then used to obtain the WLAV estimator for the system states. The paper [7] addresses the application of interior point methods to the Weighted Least Absolute Value state estimation problem. The IRLAV [8] method is almost the same as WLAV, except that the weights are routinely changed to fit new conditions during the iterations based on the measurement residuals. A fast decoupled WLAV state estimator with a constant Jacobian matrix realized through a few assumptions has been developed [9] and the technique, even faster, has generated an estimate which reflected the impact of assumptions made.

The line flow and bus voltage magnitude load flow model presented in (10) has been used in this paper to develop a similar SE model which has been solved using WLS technique by applying PSO and WIPSO algorithms. This method tends to avoid most of the factorization related matrix manipulation problems mentioned so far. 
Presence of bad data has a substantial impact over the quality of the estimate generated by the least squares based estimator and therefore special techniques have been needed to identify them and quantify their effects. A linear recursive bad data identification technique based on power system decomposition has been presented in (11). Neural network based filter has been applied for bad data detection and identification in(12) in which once trained, the filter rapidly identifies most measurement errors simultaneously by comparing the square difference of raw measurements and their corresponding estimated values with some given thresholds. Bad data prefiltering using wavelet transform has been presented in (13) and this approach identifies and filters out the bad data even before the state estimation algorithm estimates the system state. An identification algorithm based on the largest normalized residual considering statistical correlation among the measurements is presented in (14). As the proposed method here uses a constant Jacobian, unlike the conventional WLS estimator, the impact of bad measurements over the estimate has been considerably reduced and hence it doesn't require a separate algorithm to filter out the bad measurements. Over the past few decades soft computing algorithms have been playing a major role in solving optimization problems. Evolutionary programming algorithms are promising from the point of view of their capability to evade local maxima and minima. Out of the many evolutionary algorithms PSO has been widely used from the point of view of assured convergence and programming flexibility. PSO algorithm has been successfully implemented for solving the problem of SE in spite of the apprehensions such as larger computational time etc (15). In this work a line flow based WLAV state estimation problem has been formulated and it has been solved through BBO and WIPSO techniques in the absence as well as the presence of bad measurements for various standard IEEE test systems.

\subsection{Conventional WLAV State Estimation}

\section{Problem Formulation}

The WLS estimator is not a robust one because of its quadratic objective function. Therefore, an estimator involving non-quadratic objective function is used. This estimator offers a more robust estimation, which is obtained by minimising

$$
\begin{aligned}
& J=\left[\operatorname{diag}\left(R^{-1}\right)\right]^{T}|z-h(x)| \\
& =\sum_{j=1}^{n z}\left|z_{j}-h_{j}(x)\right| / \sigma_{j}{ }^{2}
\end{aligned}
$$

Since the above objective minimises the absolute value of the error weighted by the measurement accuracy $\sigma_{j}{ }^{-2}$, it is commonly called as the WLAV estimator.

The objective of Eq. (1) is reformulated using LP in order to solve the WLAV problem:

Minimise $J=\left[\operatorname{diag}\left(R^{-1}\right)\right]^{T}[\gamma+\eta]$

Subject to

$$
\begin{aligned}
& H \Delta x+\gamma-\eta=\Delta z \\
& \gamma, \eta \geq 0
\end{aligned}
$$

A SE solution is obtained by solving the LP problem given by Eq. (3) iteratively for $x$ until $\Delta x$ is sufficiently small. This method is highly inefficient, as it requires large computer memory and involves the time consuming LP technique, which itself is an iterative process and hence not suitable for real time applications. However this algorithm is robust and stable in the sense that it has the inherent feature of rejecting bad measurements by interpolating only $n s$ among the $n z$ measurements and free from ill-conditioning due to the effect of wide assignment of weighting factors and avoidance of factorisation and multiplication of several matrices. In this paper an attempt has been made to increase the computational efficiency of the robust WLAV technique through linearization.

\subsection{Proposed Method}

The real and reactive bus powers as a function of real line flows, reactive line flows and $\mathrm{V}_{\mathrm{m}}{ }^{2}$ can be written as

$P_{i}=\sum_{j=1}^{n l} A_{i j} p_{j}-\sum_{j=1}^{n l} A_{i j}^{\prime} l_{j}$

$Q_{i=} \sum_{j=1}^{n l} A_{i j} q_{j}-\sum_{j=1}^{n l} A_{i j} m_{j}$

Treating $\mathrm{P}, \mathrm{Q}$ and $\mathrm{V}_{\mathrm{m}}{ }^{2}$ as state variable[x], the measurement set $[\mathrm{Z}]$ can be represented as

$[Z]=[f(x)]$

Where 
$[Z]=\left[P, Q, p, q, V^{2}\right]^{T}$

The WLAV objective function can be written as

$\operatorname{Min} \varphi=\sum_{i=1}^{n m} w i[Z i-f i(x)]$

The above equation does not include line capacitances and shunt susceptances and hence it is inadequate to estimate the system state. However the problem can be made solvable if constraint equations including branch voltage drop and phase angle drop are considered. These constraints can be represented as

$h(x)=2 R p+2 X q-\left(\Lambda A_{1+}^{T}+A_{1-}^{T}\right) V^{2}=0$

$g(x)=C X p-C R q-C \alpha=0$

The constrained optimization problem of equations 7,8 and 9 can be formulated as a linear programming problem as

$\operatorname{Min} \varphi=\sum_{i=1}^{n m} w i\left[S i^{\prime}-S i^{\prime \prime}\right]$

Subject to

A. $\Delta x+S^{\prime}-S^{\prime \prime}=Z-f\left(x^{0}\right)$

$H . \Delta x=-h\left(x^{0}\right)$

$G \cdot \Delta x=-\mathrm{g}\left(\mathrm{x}^{0}\right)$

Where

$\mathrm{A}, \mathrm{H}$ and $\mathrm{G}$ are the jacobian matrices formed by partially differenting $\mathrm{f}(\mathrm{x}), \mathrm{h}(\mathrm{x})$ and $\mathrm{g}(\mathrm{x})$ with respect to $\mathrm{x}$.

$\Delta \mathrm{x}$ is the state correction vector

S' and S' are the slack variable vectors.

The above LP problem can be solved iteratively for $\mathrm{x}$ till the algorithm converges. It is to be noted that the jacobian matrices $\mathrm{A}, \mathrm{H}$ and $\mathrm{G}$ are constant matrices that require to be computed only at the beginning of the iterative process. However RHS vectors $\mathrm{f}(\mathrm{x}), \mathrm{g}(\mathrm{x}) \mathrm{h}(\mathrm{x})$ must be recomputed during iterative process.

\subsubsection{Introduction of BBO}

Biography is the study of distribution of species in nature. The species migrate from one island to exceptional islands which have good geographical condition. Every island is technically referred to as as habitat. The geographical areas are good geared up for extra species which might be named as high Habitat Suitability Index (HSI). Each habitat has unique features that characteristics habitability is known as Suitability Index Variables (SIV). The SIV of a habitat represents the rainfall, land discipline and temperature. The habitat stays in low HSI, the species reside in that habitat will tend to extinct. The emigration of species occur such habitats. In a similar fashion, the immigration additionally occurs when the habitat have immoderate HSI. The mathematical units of biogeography explains how species emigration and immigration within the habitats. The BBO algorithm is developed headquartered on the mathematics of biogeography. It really works established on two mechanisms named as migration and mutation. BBO is similar to the other population based optimization procedures. An island or a habitat and HSI are analogous to single population and fitness value in Genetic Algorithm (GA) respectively. Identical of these algorithms, BBO shares the information between the habitats. GA solutions die at the end of each generation. But BBO and PSO solutions survive forever. An identical group solution is clustered in PSO and not in GA and BBO.

\subsubsection{BBO Algorithm}

The BBO algorithm for LF based SE problem is described as follows.

1. Read the system data.

2. Initialize the BBO parameters like probability modification index, mutation rate, minimum and maximum value of emigration and immigration rates and Elite parameters.

3. Start the generation.

4. For each habitat, evaluate the objective function using equation (10).

5. If $\mathrm{G}<$ maximum generation and best fit $\neq 1$, otherwise go to step 13 .

6. Sort habitats based on its HSI. Keep the best HIS habitats (Elite HSI) and corresponding habitat (Elite Habitat) for the next generation.

7. For each habitat, map the immigration rate $\lambda$ and emigration rate $\mu$.

8. Probabilistically use $\lambda$ and $\mu$ to modify each non elite habitat.

9. Perform the mutation.

10. Evaluate the fitness of each new habitat and sort.

11. Replace the worst habitats with the Elite habitats

12. set $\mathrm{t}=\mathrm{t}+1$ and go to step 5

13. Store the best Habitat. 


\subsubsection{WIPSO Algorithm}

The WIPSO algorithm for LF based SE problem is described as follows.

1. Choose the population size, the number of generations, $\mathrm{W}_{\min }, \mathrm{W}_{\max }, \mathrm{C}_{1 \min }, \mathrm{C} 1 \mathrm{max}, \mathrm{C} 2 \mathrm{~min}, \mathrm{C} 2 \mathrm{max}$, pbest, gbest.

2. Initialize the velocity and position of all particles randomly, ensuring that they are within limits.

Here the individuals represent the real and reactive power flows and bus voltage magnitudes.

3. Set the generation counter $\mathrm{t}=1$.

4. Evaluate the fitness for each particle using equation (10) according to the objective function.

5. Compare the particle's fitness function with its $P_{\text {best } i}$. If the current value is better than $P_{\text {best } i}$, then set $P_{\text {best } i}$ is equal to the current value. Identify the particle in the neighborhood with the best success so far and assign it to Gbest.

6. Update the value of the weight factor using equation (15).

7. Update velocity by using the global best and individual best of the particle.

8. Update position by using the updated velocities. Each particle will change its position.

9. If the stopping criteria is not satisfied set $\mathrm{t}=\mathrm{t}+1$ and go to step 4.Otherwise stop.

\section{Simulation and Results}

The proposed LFBSE problem have been solved using BBO and WIPSO techniques by selecting a habitat size of 20 habitat modification probability $=1$, Immigration probability bounds per gene $=\{0,1\}$, step size for numerical integration of probabilities $=1$, maximum immigration and migration rates of each island $=1$ and mutation probability $=0.1$, Maximum Generation $=100$. It has been verified on standard IEEE 14, 30 and 57 bus test systems. The measurement vector has been generated by way of adding a small percentage of noise to the values obtained from the Newton Raphson load flow. Bus voltage magnitudes at the load buses and real and reactive power flows through the lines were taken as state variables. All the line flows, bus power injections and bus voltage magnitudes at the even numbered buses were considered in the measurement set to achieve necessary redundancy. To study the performance of the algorithm in the presence as well as absence bad measurements, in each of the measurement set, 5, 10 and 15 number of bad measurements were introduced randomly. The performance of the algorithm has been validated by evaluating the results of the proposed method against the results obtained using standard WLS state estimation and LFWLAV State Estimation. The algorithms were tested with a flat start and a convergence tolerance of 0.0001 . Three performance indices are defined to validate the performance of the proposed technique. They are $\Delta \mathrm{V}_{\mathrm{rms}}, \Delta \mathrm{p}_{\mathrm{rms}}, \Delta \mathrm{q}_{\mathrm{rms}}$.

$$
\begin{aligned}
\Delta \text { Vrms } & =\sqrt{\frac{1}{n b}} \sum_{i}^{n b}\left(V_{i}^{t}-V_{i}\right)^{2} \\
\Delta p_{r m s} & =\sqrt{\frac{1}{n l}} \sum_{i}^{n l}\left(P_{i}^{t}-P_{i}\right)^{2} \\
\Delta q_{r m s} & =\sqrt{\frac{1}{n l}} \sum_{i}^{n l}\left(q_{i}^{t}-q_{i}\right)^{2}
\end{aligned}
$$

Tables 1, 2 and 3 compare the performance of the proposed method with WLAV and LFWLAV estimation algorithm in terms of the performance indices defined in 1,2 and 3 and NET. The performance of the algorithm

\begin{tabular}{|c|c|c|c|c|c|}
\hline Measurements & Method & $\Delta \mathrm{Vrms}$ & $\Delta$ Prms & $\Delta$ Qrms & NET in $\mathrm{ms}$ \\
\hline \multirow{4}{*}{ 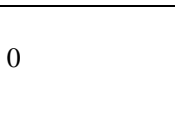 } & WLAV & 0.1406 & 0.1351 & 0.1643 & 211 \\
\hline & LFWLAV & 0.0883 & 0.1103 & 0.111 & 136 \\
\hline & LFWLAV-BBO & 0.0789 & 0.1081 & 0.1086 & 148 \\
\hline & LFWLAV-WIPSO & 0.0846 & 0.1088 & 0.1091 & 143 \\
\hline \multirow{4}{*}{5} & WLAV & 0.1405 & 0.1286 & 0.1631 & 210 \\
\hline & LFWLAV & 0.0882 & 0.1074 & 0.1094 & 136 \\
\hline & LFWLAV-BBO & 0.0764 & 0.1027 & 0.1074 & 149 \\
\hline & LFWLAV-WIPSO & 0.0837 & 0.1029 & 0.1081 & 144 \\
\hline \multirow{4}{*}{10} & WLAV & 0.1363 & 0.1277 & 0.1573 & 212 \\
\hline & LFWLAV & 0.0635 & 0.1034 & 0.1083 & 137 \\
\hline & LFWLAV-BBO & 0.0513 & 0.1019 & 0.1071 & 149 \\
\hline & LFWLAV-WIPSO & 0.0509 & 0.1027 & 0.108 & 144 \\
\hline \multirow{4}{*}{15} & WLAV & 0.1349 & 0.1215 & 0.138 & 212 \\
\hline & LFWLAV & 0.0246 & 0.1027 & 0.1078 & 137 \\
\hline & LFWLAV-BBO & 0.0176 & 0.1011 & 0.1053 & 149 \\
\hline & LFWLAV-WIPSO & 0.0179 & 0.1022 & 0.1065 & 146 \\
\hline
\end{tabular}
is also illustrated through bar charts in Fig 1 to 12.

Table 1: Results for IEEE 14 Bus Systems 
Line Flow Based WLAV State Estimation using BBO and WIPSO Techniques

Table 2: Results for IEEE 30 Bus Systems

\begin{tabular}{|l|l|l|l|l|l|}
\hline Measurements & Method & $\Delta$ Vrms & $\Delta$ Prms & $\Delta$ Qrms & NET in ms \\
\hline \multirow{5}{*}{0} & WLAV & 0.1742 & 0.3824 & 0.2117 & 468 \\
\cline { 2 - 6 } & LFWLAV & 0.0755 & 0.2173 & 0.1325 & 189 \\
\cline { 2 - 6 } & LFWLAV-BBO & 0.0639 & 0.2127 & 0.1304 & 202 \\
\cline { 2 - 6 } & LFWLAV-WIPSO & 0.0646 & 0.2133 & 0.1313 & 197 \\
\hline \multirow{5}{*}{5} & WLAV & 0.0833 & 0.3794 & 0.2109 & 469 \\
\cline { 2 - 6 } & LFWLAV & 0.0397 & 0.2159 & 0.1319 & 188 \\
\cline { 2 - 6 } & LFWLAV-BBO & 0.0303 & 0.2121 & 0.1295 & 201 \\
\cline { 2 - 6 } & LFWLAV-WIPSO & 0.0309 & 0.2129 & 0.1304 & 198 \\
\hline \multirow{5}{*}{15} & WLAV & 0.0609 & 0.3756 & 0.2099 & 469 \\
\cline { 2 - 6 } & LFWLAV & 0.0328 & 0.2138 & 0.1311 & 189 \\
\cline { 2 - 6 } & LFWLAV-BBO & 0.0286 & 0.2112 & 0.1287 & 202 \\
\cline { 2 - 6 } & LFWLAV-WIPSO & 0.0298 & 0.2117 & 0.1296 & 198 \\
\hline & WLAV & 0.0454 & 0.3743 & 0.2081 & 469 \\
\cline { 2 - 6 } & LFWLAV & 0.0283 & 0.213 & 0.1305 & 189 \\
\cline { 2 - 6 } & LFWLAV-BBO & 0.0216 & 0.2003 & 0.1283 & 202 \\
\cline { 2 - 6 } & LFWLAV-WIPSO & 0.022 & 0.2012 & 0.1289 & 199 \\
\hline
\end{tabular}
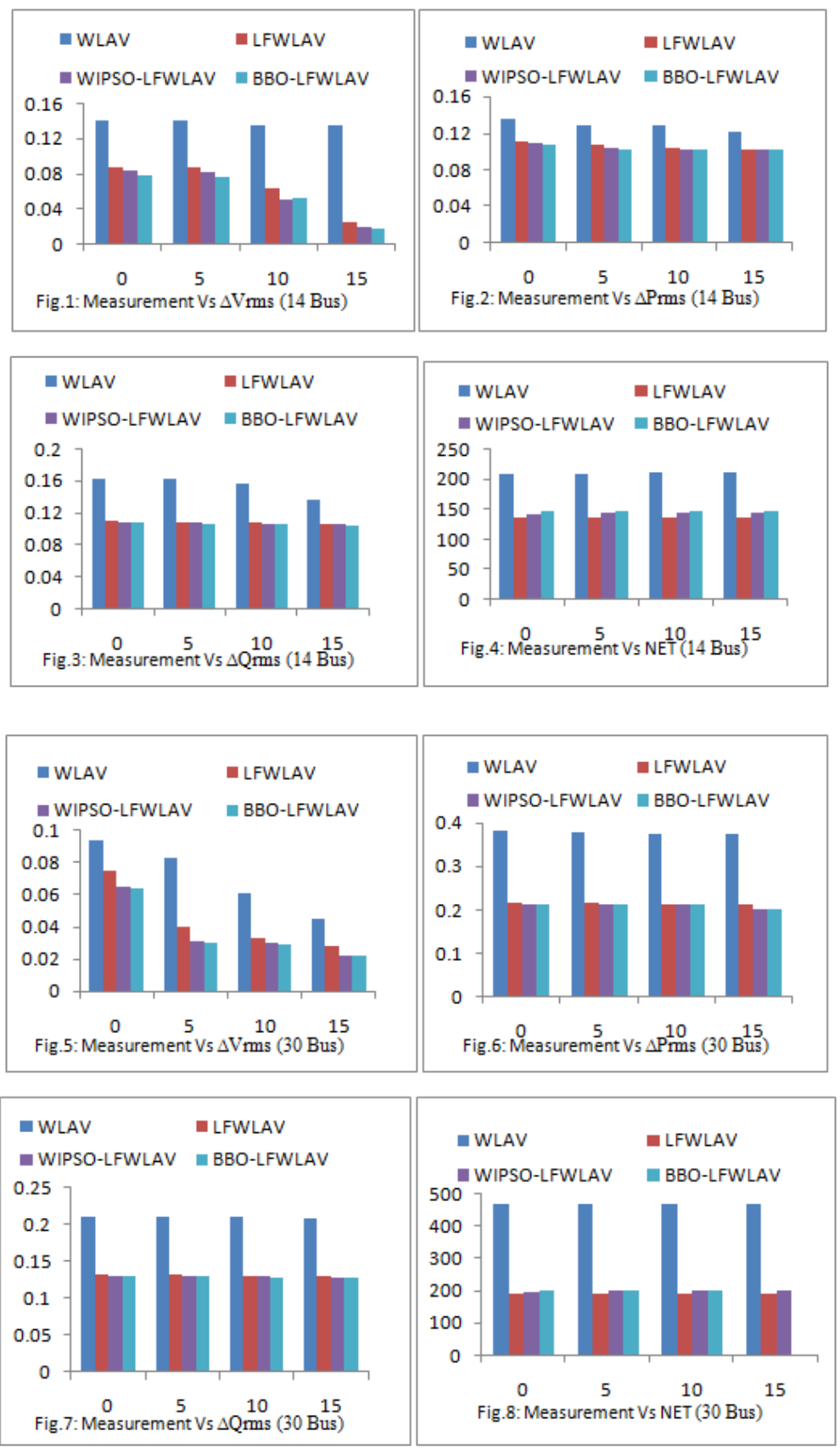
Table 3: Results for IEEE 57 Bus Systems

\begin{tabular}{|l|l|l|l|l|l|}
\hline \multicolumn{5}{|l|}{} \\
\hline Measurements & Method & $\Delta$ Vrms & $\Delta$ Prms & $\Delta$ Qrms & NET in ms \\
\hline 0 & WLAV & 0.0791 & 0.2579 & 0.1346 & 711 \\
\cline { 2 - 6 } & LFWLAV & 0.0288 & 0.1173 & 0.1091 & 233 \\
\cline { 2 - 6 } & LFWLAV-BBO & 0.0276 & 0.1148 & 0.107 & 257 \\
\cline { 2 - 6 } & LFWLAV-WIPSO & 0.0282 & 0.1152 & 0.1077 & 253 \\
\hline 5 & WLAV & 0.0788 & 0.2553 & 0.1332 & 709 \\
\cline { 2 - 6 } & LFWLAV & 0.0283 & 0.1164 & 0.1083 & 234 \\
\cline { 2 - 6 } & LFWLAV-BBO & 0.0254 & 0.1139 & 0.1053 & 257 \\
\cline { 2 - 6 } & LFWLAV-WIPSO & 0.0262 & 0.1145 & 0.1061 & 254 \\
\hline 10 & WLAV & 0.0782 & 0.2527 & 0.132 & 709 \\
\cline { 2 - 6 } & LFWLAV & 0.0272 & 0.1158 & 0.1071 & 232 \\
\cline { 2 - 6 } & LFWLAV-BBO & 0.0246 & 0.1133 & 0.1041 & 258 \\
\cline { 2 - 6 } & LFWLAV-WIPSO & 0.0252 & 0.1138 & 0.1048 & 254 \\
\hline 15 & WLAV & 0.0774 & 0.2502 & 0.1313 & 710 \\
\cline { 2 - 6 } & LFWLAV & 0.0258 & 0.115 & 0.106 & 232 \\
\cline { 2 - 6 } & LFWLAV-BBO & 0.024 & 0.1124 & 0.103 & 259 \\
\cline { 2 - 6 } & LFWLAV-WIPSO & 0.0246 & 0.1131 & 0.1038 & 254 \\
\hline
\end{tabular}
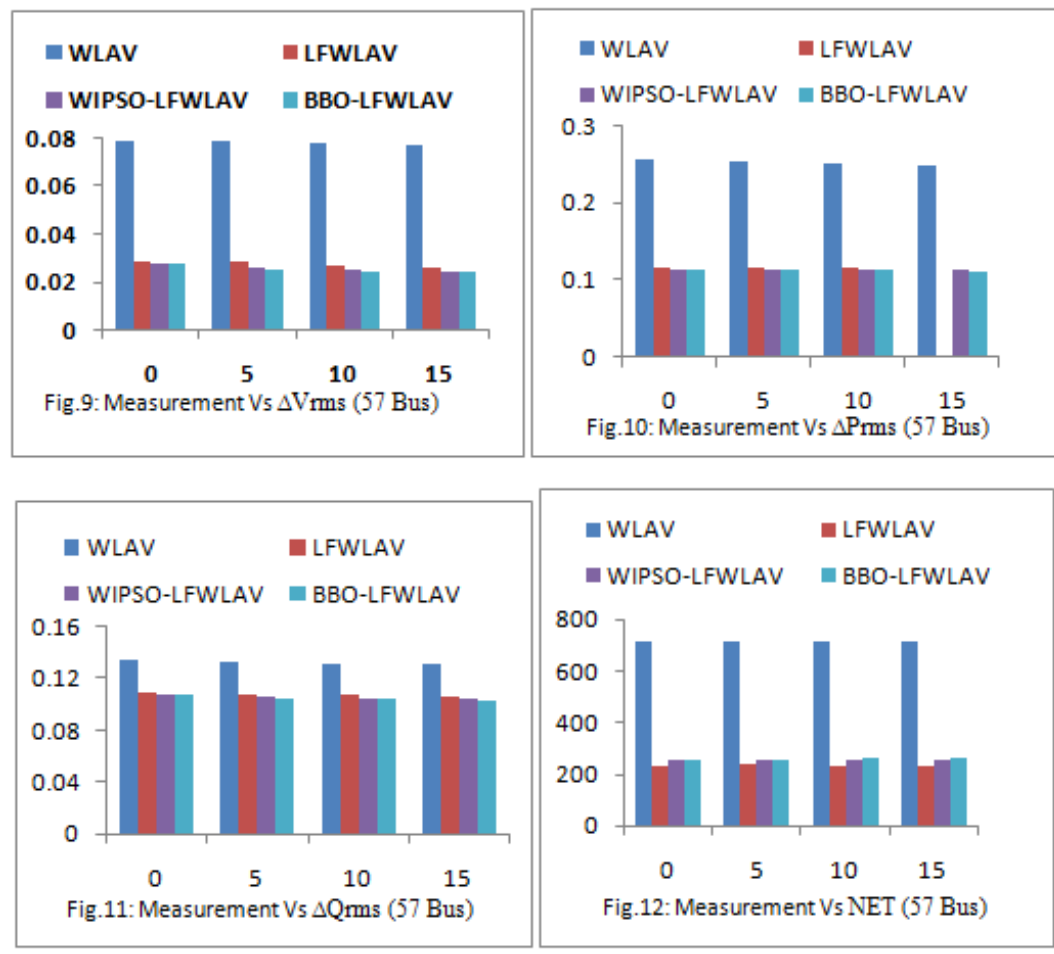

\section{Conclusion}

A novel line flow based state estimation technique which results in the formation of constant jacobian matrix has been presented in this paper and it has been solved through WLAV method. BBO and WIPSO technique had been implemented for solving the LFBWLAV problem in the presence as well as absence of bad measurements. The results point out that the normalized value of the error between the actual values and estimated values of the state variables is noticeably lesser in the case of proposed method when solved using BBO and WIPSO than that of the conventional WLAV and LFBWLAV techniques. Further there is a marginal increase in computation time due to the heuristic search nature of BBO algorithm still as the estimated system state is closer to the actual system state in the proposed method; BBO method is very much suitable for security studies of power systems.

\section{References}

[1] Schweppe.F.C, Wildes. J., "power system static-state estimation, Part I: Exact Model", IEEE Trans. on Power Apparatus and Systems, Vol.PAS-89, Issue: 1, 1970, pp. 120-125.

[2] Schweppe. F.C.; Rom.D.B, "power system static-state estimation, Part II: Approximate Model”, IEEE Trans. on Power Apparatus and Systems, Vol.PAS-89, Issue: 1, 1970, pp. 125-130.

[3] Schweppe. F.C., "power system static-state estimation, Part III: Implementation", IEEE Trans. on Power Apparatus and Systems, Vol.PAS-89, Issue: 1, 1970, pp. 130-135. 
[4] Larson.R, Tinney.W.F, Hajdu.L.P and Piercy.D.S, "State Estimation in Power Systems Part II: Implementation and applications", IEEE Trans. on Power Apparatus and Systems, Vol.PAS-89, Issue: 3, 1970, pp. 353-363.

[5] Whei-Min Lin and Jen-Hao Teng, "A new transmission fast-decoupled state estimation with equality constraints", Electric Power and Energy Systems, Vol. 20, No.7, 1998, pp.489-493.

[6] M.K. Celik and A. Abur, “A robust WLAV state estimator using transformation”, IEEE Trans. on Power Systems, 1992, 7(1): 106112.

[7] H. Singh F.L. Alvarado, "Weighted Least Absolute Value State Estimation using Interior Point Method", IEEE Transactions on Power Systems, 1994, 9(3).

[8] R.A. Jabr and B.C.Pal, "Iteratively reweighted least-square implementation of the WLAV state estimation method", IEE Proc. Gener. Transm. Distrib., 2004, 151(1): 103-108.

[9] R.Neela and R.Ashokumar, "A robust decoupled WLAV state estimation for power system", International Journal of Engineering Science and Technology, 2010, 2(8): 3590-3596.

[10] P.Yan, A.Sekar, "Study state analysis of power system having multiple Facts devices using line flow based equations", IET Proceedings-Generation Transmission and Distribution, 2005, Vol.152, Issue 1, pp.31-39.

[11] B.M.Zhang, S.Y.Wang and N.D.Xiang, "A linear recursive bad data identification method with real time application to power system state estimation", IEEE Trans. on Power Systems, 1992, Vol.7, No.3, pp.1378-1385.

[12] H.Salehfar, R.Zhao," A neural network preestimation filter for bad-data detection and identification in power system state estimation”, Electric Power System Research, 1995, Vol.34, pp.127-134.

[13] D.Singh, R.K.Misra, V.K.Singh R.K.Pandey, “Bad data pre-filter for state estimation”, Electric Power and Energy Systems, 2010, Vol. 32, pp.1165-1174.

[14] Eduardo Caro, Antonio J.Conejo, Robert Minguez, Marija Zima and Goran Anderson, "Multiple bad data identification considering measurement dependencies”, IEEE Trans. IEEE Trans. on Power Systems, 2011, Vol.26, No.4, pp.1953-1961

[15] D.H.Tungadio, B.P.Numbi, M.W.Siti, A.A.Jimoh, "Particle swarm optimization for power system state estimation", Neurocomputing, 2015, Vol.148, pp. 175-180. 